\title{
Second-order method for Parabolic Volterra Integral Equations with Crank-Nicolson Method
}

\author{
Ali Filiz
}

\begin{abstract}
In this paper using Crank-Nicolson method we investigate convergence properties of time discretization of Parabolic Volterra integral equations are studied. The rectangle, the trapezoidal and Simpson's rules applied for these equations. The integral is approximated in each case by the quadrature rule. The main idea of this paper quadrature rules based a fewer points, reducing the number of time levels at which the data need to be saved. We consider time step methods based on Crank-Nicolson method and combined with the appropriate quadrature rules.
\end{abstract}

\section{INTRODUCTION}

A common numerical method for solving all types of partial differential equations is the finite difference method (see [1], [10] or [4]). The simplest implicit method was given first by [6]. Another implicit method is the CrankNicolson $(\mathrm{CN})$ method ([2]). In this paper we consider the parabolic partial differential equation including a Volterra integral term which is called the parabolic Volterra integro-differential equation (PVIDE) ([7] called these kind of equations integroparabolic equations of Volterra type).

The goal of this paper is to present some methods for the numerical solution of parabolic Volterra integro-differential equations ([7], [8] or [9]). The linear PVIDEs was given by [11] and basic methods for numerical solution of this equation were introduced by [3] using the backward-Euler and CN methods. Various numerical methods (Crank-Nicolson and their mixed methods) can be used for linear PVIDEs and their errors are illustrated in Section 2.

In [5], we considered the numerical solution of Parabolic Volterra integral equations with the backward-Euler method using the rectangle, the trapezoidal rules for finding $u(x, t)$ with the quadrature rule for integral term. Whereas in this paper we introduce the numerical treatment of parabolic Volterra equations using Crank-Nicolson scheme for finding $u(x, t)$ with the

2010 Mathematics Subject Classification. The form is: Primary: 65M06; Secondary: $45 \mathrm{D} 05$.

Key words and phrases. Parabolic Volterra integro-differential(integral) equation, Crank-Nicolson (CN) scheme, mixed rule, quadrature rule. 
finite difference method.

$$
\frac{\partial u}{\partial t}(x, t)=\kappa \frac{\partial^{2} u}{\partial x^{2}}(x, t)+\lambda \int_{0}^{t} K(t, s) B u(x, s) \mathrm{d} s+S(x, t),
$$

where $B$ is a second order partial differential operator and smooth.

\section{Mixed Crank-Nicolson Method For PARABOlic Volterra EQUations}

In [3] and [9] deal with stability and convergence results for two different time discretizations of (1), based on the backward-Euler and Crank-Nicolson methods respectively. However, in their paper the numerical examples are not given. In this paper the time and space discretization are studied with example. We have used MATHEMATICA, MATLAB and FORTRAN programs for symbolic and numerical calculations. Especially, time discretization is worked in the treatment of the integral term. In this section we shall investigate the numerical solution of equation (1) with combined Simpson's rule, trapezoidal rule and rectangle rule. In the Crank-Nicolson scheme the time discretization of equation (1) is

$$
\begin{aligned}
\frac{U_{n, j}-U_{n, j-1}}{k} & =\frac{\kappa}{2 h^{2}}\left\{\left(U_{n-1, j}-2 U_{n, j}+U_{n+1, j}\right)\right. \\
& \left.+\left(U_{n-1, j-1}-2 U_{n, j-1}+U_{n+1, j-1}\right)\right\} \\
& +\lambda \sum_{j=0}^{n-1} \omega_{n j} K\left(\left(n-\frac{1}{2}\right) k, j k\right) B U(i h, j k) \\
& +S\left(i h,\left(n-\frac{1}{2}\right) k\right), \quad n \geq 1,
\end{aligned}
$$

where the sum on the RHS is a quadrature approximation to the integral term in (1) with $t_{n}=\left(n-\frac{1}{2}\right) k$. If we take $\kappa=1.0$ and $\lambda=-1.0$ we obtain the following form

$$
\begin{aligned}
\frac{U_{n, j}-U_{n, j-1}}{k} & =\frac{1}{2 h^{2}}\left(U_{n-1, j}-2 U_{n, j}+U_{n+1, j}\right) \\
& +\frac{1}{2 h^{2}}\left(U_{n-1, j-1}-2 U_{n, j-1}+U_{n+1, j-1}\right) \\
& -\sum_{j=0}^{n-1} \omega_{n j} K\left(\left(n-\frac{1}{2}\right) k, j k\right) B U(i h, j k) \\
& +S\left(i h,\left(n-\frac{1}{2}\right) k\right), \quad n \geq 1 .
\end{aligned}
$$

Hence the weights $\omega_{n j}$ in the quadrature rule correspond to

$$
\int_{0}^{\left(n-\frac{1}{2}\right) k} g(s) \mathrm{d} s \approx \sum_{j=0}^{n-1} \omega_{n j} g(j k) .
$$


Let $l=l(n)$ be the largest nonnegative integer such that $2 l k_{1}<n k$, and write the interval of integration as $\left[0,2 l k_{1}\right] \cup\left[2 l k_{1},(n-1) k\right] \cup[(n-$ $\left.1) k,\left(n-\frac{1}{2}\right) k\right]$. First we use Simpson's rule with step-size $k_{1}$, secondly the trapezoidal rule with step-size $k$, and lastly a single rectangle rule with stepsize $\frac{k}{2}$. Therefore the quadrature approximation is

$$
\begin{aligned}
& \int_{0}^{\left(n-\frac{1}{2}\right) k} g(s) \mathrm{d} s \approx \frac{1}{3} k_{1}\left[g(0)+4 g\left(k_{1}\right)+2 g\left(2 k_{1}\right)+\cdots\right. \\
&\left.\cdots+4 g\left((2 l-1) k_{1}\right)\right] \\
&+\left(\frac{1}{3} k_{1}+\frac{1}{2} k_{1}\right) g\left(2 l k_{1}\right) \\
&+k\left[g\left(2 l k_{1}+k\right)+\cdots+g((n-1) k)\right] .
\end{aligned}
$$

If $l=0$ there is no Simpson's rule interval, and if $n=2 l m+1$ there is no trapezoidal rule. Hence equation (4) is modified accordingly. If $n k$ is bounded by $T$ the truncation error is of $O\left(k_{1}^{4}+k_{1} k^{2}+k^{2}\right)$. Thus a truncation error of $O\left(k^{2}\right)$, and hence consistency with the Crank-Nicolson scheme is, ensured if $k_{1}=O\left(k^{1 / 2}\right)$.

\subsection{How to Use CN Scheme.}

$$
\omega_{j v}= \begin{cases}\omega_{v}^{1}=k_{1}, & \text { when } j \equiv 0(\bmod m), \\ \omega_{v}^{1}=0, & \text { when } j \not \equiv 0(\bmod m), j-v>m, \\ \omega_{v}^{2}=k, & \text { otherwise. }\end{cases}
$$

The weights of the scheme (4) are representable in the form (5), with $\omega_{j}^{1}$ and $\omega_{j}^{2}$ both independent of $n$, and $i(j)$ the smallest multiple of $\frac{2 k_{1}}{k}=2 m$ which is greater than $j$. The weights $\omega_{j}^{1}$ are Simpson's rule weights, and have the values $\frac{k_{1}}{3}, \frac{4 k_{1}}{3}, \frac{2 k_{1}}{3}$ or zero according to whether $j$ is zero, an odd multiple of $m$, a nonzero multiple of $2 m$, or not a multiple of $m$. The weights $\omega_{j}^{2}$, which are those that apply when $n$ is near $j$, have the value $k$, unless $j$ is an even multiple of $m$, in which case $\omega_{j}^{2}$ has the value $\frac{k_{1}}{3}+\frac{k_{1}}{2}$ for $j \neq 0$, and $\frac{k_{1}}{2}$ for $j=0$.

2.2. How to apply $\mathbf{C N}+$ Trapezoidal rule. We will use the following example for our numerical calculations.

Example 2.1. Consider the initial-boundary value problem

$$
\frac{\partial u}{\partial t}(x, t)=\kappa \frac{\partial^{2} u}{\partial x^{2}}(x, t)+\lambda \int_{0}^{t} \exp (-(t-s)) u(x, s) \mathrm{d} s,
$$


for $0 \leq t \leq T, 0 \leq x \leq \pi$, with $u(x, 0)=\sin x, u(0, t)=u(\pi, t)=0$, which has the exact solution (see [5])

$$
u(x, t)=\exp (-\alpha t)\left(\cos \beta t+\frac{1-\alpha}{\beta} \sin \beta t\right) \sin x,
$$

where $\alpha=\frac{\kappa+1}{2}$ and $\beta=\frac{\sqrt{-\kappa^{2}+2 \kappa-1-4 \lambda}}{2}$.

Taking $\kappa=1$ in the equation (7) we have the solution

$$
u(x, t)=\exp (-t) \cosh \sqrt{\lambda} t \sin x, \text { for } \lambda>0 .
$$

If we put $\kappa=1$ and $\lambda=-1$ in (7) we obtain the solution

$$
u(x, t)=\exp (-t) \cos t \sin x
$$

In this method we apply the CN scheme for the parabolic term and the trapezoidal rule for the integral term. In equation (4) the upper limit of the integral term is $\left(n-\frac{1}{2}\right) k$ which requires midpoint evaluation. It is clear that the CN scheme gives $O\left(k^{2}\right)$ in time discretization and applying the trapezoidal rule for integral term gives $O\left(k^{2}\right)$. Consequently we expect to get order $k^{2}$ in Example 2.1.

\section{$\mathrm{CN}+$ trapezoidal rule:}

Below we will explain the integral term calculation according to trapezoidal rule.

$$
\int_{0}^{\left(j+\frac{1}{2}\right) k} \exp (-t+s) u(x, s) \mathrm{d} s=\exp (-t) \int_{0}^{\left(j+\frac{1}{2}\right) k} \underbrace{\exp (s) u(x, s)}_{g(s)} \mathrm{d} s .
$$

First of all, if $j=0$ we get

$$
\begin{aligned}
\exp (-k / 2) \int_{0}^{\frac{k}{2}} g(s) \mathrm{d} s & \approx \exp (-k / 2) \frac{k}{2}\left\{\frac{1}{2} g(0)+\frac{1}{2} g\left(\frac{k}{2}\right)\right\} \\
& \approx \exp (-k / 2) \frac{k}{4}\left\{g(0)+\frac{g(0)+g(k)}{2}\right\} \\
& =\exp (-k / 2) \frac{k}{8}\{3 g(0)+g(k)\}
\end{aligned}
$$

The last term involving $g(k)$ is transferred to the implicit equations on the left. 
Second, if we take $j=1$ we find

$$
\begin{aligned}
& \exp (-3 k / 2) \int_{0}^{\frac{3 k}{2}} g(s) \mathrm{d} s= \\
& \quad=\exp (-3 k / 2)\left\{\int_{0}^{\frac{k}{2}} g(s) \mathrm{d} s+\int_{k / 2}^{k} g(s) \mathrm{d} s+\int_{k}^{\frac{3 k}{2}} g(s) \mathrm{d} s\right\} \\
& \quad \approx \exp (-3 k / 2) \frac{k}{8}\{4 g(0)+7 g(k)+g(2 k)\} .
\end{aligned}
$$

Again the last term is transferred to the left. If we take $j=m-1$

$$
\left.\begin{array}{rl}
\exp (-(m-1 / 2) k) \int_{0}^{(m-1 / 2) k} g(s) \mathrm{d} s \approx \\
\approx \exp (-(m-1 / 2) k) & \{\underbrace{\int_{0}^{(m-3 / 2) k} g(s) \mathrm{d} s}_{\text {sum }}+ \\
+\frac{k}{8}((g(m-2) k)+7 g((m-1) k+g(m k))
\end{array}\right\},
$$

and the last term is transferred to the left.

\begin{tabular}{|c|c|c|c|}
\hline$t=2.0$ & & $k=0.02$ & $k=0.01$ \\
\hline$x$ & EXACT & ERROR 1 & ERROR 2 \\
\hline 0.31 & -0.01740 & $0.128816 \mathrm{E}-05$ & $0.297192 \mathrm{E}-06$ \\
\hline 0.63 & -0.03310 & $0.245022 \mathrm{E}-05$ & $0.565293 \mathrm{E}-06$ \\
\hline 0.94 & -0.04556 & 0.337244E-05 & $0.778060 \mathrm{E}-06$ \\
\hline 1.26 & -0.05356 & $0.396454 \mathrm{E}-05$ & $0.914664 \mathrm{E}-06$ \\
\hline 1.57 & -0.05632 & $0.416857 \mathrm{E}-05$ & $0.961734 \mathrm{E}-06$ \\
\hline$\|$ error $\|_{2}$ & & $4.168565 \mathrm{E}-05$ & $9.617344 \mathrm{E}-06$ \\
\hline
\end{tabular}

We use these formulae for $\mathrm{CN}+$ trapezoidal rule and find truncation error $O\left(k^{2}\right)$. The numerical results are given in Table 1 .

CN + TRAPEZOIDAL RULE

SHOWING EFFECT OF TIME-STEP $k$,

FOR EXAMPLE 2.1.

TABLE 1. Table illustrates that the $\mathrm{CN}+$ trapezoidal rule has error of order $k^{2}$. 
$\mathrm{CN}+$ trapezoidal + rectangle, $\mathrm{CN}+$ Simpson + trapezoidal rule and $\mathrm{CN}$ + Simpson + trapezoidal + rectangle rule calculations can be done similarly.

In Table 2 we have used the trapezoidal rule with step-size $k$ and one rectangle rule for the last step $\frac{k}{2}$. For this calculation we also obtained accuracy of $O\left(k^{2}\right)$.

CN + TRAPEZOIDAL + RECTANGLE RULE
SHOWING EFFECT OF TIME-STEP $k$,
FOR EXAMPLE 2.1.
$h=\frac{\pi}{200}$
\begin{tabular}{|l|r|r|r|}
\hline$t=2.0$ & & $k=0.02$ & $k=0.01$ \\
\hline$x$ & EXACT & ERROR 1 & ERROR 2 \\
\hline 0.31 & -0.01740 & $0.318962 \mathrm{E}-05$ & $0.772553 \mathrm{E}-06$ \\
\hline 0.63 & -0.03310 & $0.606702 \mathrm{E}-05$ & $0.146948 \mathrm{E}-05$ \\
\hline 0.94 & -0.04556 & $0.835053 \mathrm{E}-05$ & $0.202257 \mathrm{E}-05$ \\
\hline 1.26 & -0.05356 & $0.981664 \mathrm{E}-05$ & $0.237767 \mathrm{E}-05$ \\
\hline 1.57 & -0.05632 & $0.103218 \mathrm{E}-04$ & $0.250004 \mathrm{E}-05$ \\
\hline \hline$\|$ error $\|_{2}$ & & $0.103218 \mathrm{E}-03$ & $0.250004 \mathrm{E}-04$ \\
\hline
\end{tabular}

TABLE 2. Table illustrates that the $\mathrm{CN}+$ trapezoidal rule + rectangle rule has error of order $k^{2}$.

CN + SIMPSON + TRAPEZOIDAL RULE SHOWING EFFECT OF TIME-STEP $k$, FOR EXAMPLE 2.1.

\begin{tabular}{|c|c|c|c|}
\hline$t=2.0$ & & $\begin{array}{r}k=0.05 \\
k_{1}=0.224\end{array}$ & $\begin{aligned} k & =0.025 \\
k_{1} & =0.158\end{aligned}$ \\
\hline$x$ & EXACT & ERROR 1 & ERROR 2 \\
\hline 0.31 & -0.017403636 & $0.822659 \mathrm{E}-05$ & $0.203142 \mathrm{E}-05$ \\
\hline 0.63 & -0.033103683 & $0.156479 \mathrm{E}-04$ & $0.386399 \mathrm{E}-05$ \\
\hline 0.94 & -0.045563311 & $0.215375 \mathrm{E}-04$ & $0.531833 \mathrm{E}-05$ \\
\hline 1.26 & -0.053562885 & $0.253188 \mathrm{E}-04$ & $0.625207 \mathrm{E}-05$ \\
\hline 1.57 & -0.056319350 & $0.266218 \mathrm{E}-04$ & $0.657382 \mathrm{E}-05$ \\
\hline |lerror & & ב0.266218E-03 & "0.657382E-04 \\
\hline
\end{tabular}

TABLE 3. Table illustrates that the combined CN (mixed rule) has error of order $k^{2}$. 
$\mathrm{CN}+\mathrm{SIMPSON}+$ TRAPEZOIDAL + RECTANGLE

RULE SHOWING EFFECT OF TIME-STEP $k$,

FOR EXAMPLE 2.1 .

$h=\frac{\pi}{200}$
\begin{tabular}{|l|r|r|r|}
\hline$t=2.0$ & & $k=0.05$ & $k=0.025$ \\
& & $k_{1}=0.224$ & $k_{1}=0.158$ \\
\hline$x$ & EXACT & ERROR 1 & ERROR 2 \\
\hline 0.31 & -0.017403636 & $0.201115 \mathrm{E}-04$ & $0.500248 \mathrm{E}-05$ \\
\hline 0.63 & -0.033103683 & $0.382544 \mathrm{E}-04$ & $0.951528 \mathrm{E}-05$ \\
\hline 0.94 & -0.045563311 & $0.526527 \mathrm{E}-04$ & $0.130967 \mathrm{E}-04$ \\
\hline 1.26 & -0.053562885 & $0.618969 \mathrm{E}-04$ & $0.153960 \mathrm{E}-04$ \\
\hline 1.57 & -0.056319350 & $0.650823 \mathrm{E}-04$ & $0.161884 \mathrm{E}-04$ \\
\hline \hline$\|$ error $\|_{2}$ & & $0.650823 \mathrm{E}-03$ & $0.161884 \mathrm{E}-03$ \\
\hline
\end{tabular}

TABLE 4. Table illustrates that the combined CN (mixed rule) has error of order $k^{2}$.

Tables 3, 4 illustrate the use of CN with the Simpson + trapezoidal rule, where Table 4 also has one rectangle rule step at the end. It is clear that both methods have error of $O\left(k^{2}\right)$.

2.3. Analysis of CN. In (2) we have shown that the Crank-Nicolson scheme (with $\theta=0.5$ ) gives local truncation error of $O\left(k^{2}\right)$ for our particular problem (6). In [5], we have investigated the numerical solution of problem (1) implicitly using mixed backward-Euler method with $O(k)$.

In Section 2, our main purpose was to show that the truncation error is $O\left(k^{2}\right)$ for equation (6) applying Crank-Nicolson for parabolic part and Simpson + trapezoidal + rectangle for the integral term of (6). In Table 1, we have shown that the local truncation error is $O\left(k^{2}\right)$. Secondly, we have used CN for parabolic part and the trapezoidal rule with step-size $k$ except at the last half panel when the rectangle rule applied with step-size $\frac{k}{2}$ for integral term. Then, we found order of $k^{2}$ for numerical solution of equation (6) (Table 2).

Thirdly, Simpson + trapezoidal rule was applied for integral term. In this method we start with the trapezoidal rule until $t=2 k_{1}$, then introduce Simpson rule from 0 to $2 k_{1}$ with step-size $k_{1}$ and once trapezoidal rule from $2 k_{1}$ to $2 k_{1}+\frac{k}{2}$ with step-size $\frac{k}{2}$. The calculation can be continued in a similar manner. The numerical solution of this method has been given in Table 3 . Finally, Simpson + trapezoidal + rectangle rule applied for integral term in equation (6) gave error of $O\left(k^{2}\right)$. 


\title{
3. Conclusion
}

Then, what we are expecting for this calculation? In fact, after this calculation we were expecting order of $O\left(k^{2}\right)$. In view, it seems to be true because of the truncation error for $\mathrm{CN}$ is $O\left(k^{2}+h^{2}\right)$ (for PPDE-Parabolic Partial Differential Equation) and at least the truncation error of the trapezoidal rule is $O\left(k^{2}\right)$ (for integral term). Thus, we found the expected $O\left(k^{2}\right)$ for all cases, consistent with [9].

Numerical order of convergence is also calculated:

$$
\text { Ord }=\frac{\ln \left(\text { Error }_{1}\right)-\ln \left(\text { Error }_{2}\right)}{\ln (2)} .
$$

We expect that Ord $=2$. Obtained theoretical results are confirmed by numerical experiments.

\section{REFERENCES}

[1] W.F. Ames, Numerical Methods for Partial Differential Equations, Thomas Nelson and Sons Ltd, Canada, 1969.

[2] J. Crank and P. Nicolson, A practical method for numerical evaluation of solutions of partial differential equations of the heat-conduction type, Proc. Camb. phil. Soc., 43, (1947), 50-67.

[3] J. Douglas, Numerical methods for integro-differential equations of parabolic and hyperbolic types, Numer. Math., 4, (1962), 96-102.

[4] P. DuChateau and D. Zachmann, Applied Partial Differential Equations, Harper \& Row, New York, 1989.

[5] A. Filiz, Numerical solution of some Volterra integral equations, PhD Thesis, University of Manchester, 2000.

[6] G.G. O'Brien, M. A. Hyman and S. Kaplan, A study of the numerical solution of partial differential equations, J. Math. Phys., 29, (1950), 223-251.

[7] C.V. Pao, Nonlinear Parabolic and Elliptic Equations, Plenum Press, New York, 1992.

[8] J. Prüss, Evolutionary Integral Equations and Applications, Birkhäuser Verlang, Berlin,1993.

[9] H.I. Sloan and V. Thomée, Time discretization of an integro-differential equation of parabolic type, Siam Journal of Numerical Analysis, 23, (1986), 1052-1061.

[10] G.D. Smith, Numerical Solution of Partial Differential Equations: Finite Difference Methods, Clarendon Press, Oxford, 1985.

[11] V. Volterra, Theory of Functionals and of Integro-Differential Equations, Dover, New York, 1959.

\author{
Ali Filiz \\ Department of Mathematics \\ FACUlTy of SCIENCE \\ Adnan Menderes University \\ 09010 AYDin \\ TURKEY \\ E-mail address: afiliz@adu.edu.tr
}

